\title{
Chronic pelvic pain and pelvic organ prolapse: a consequence of upright position?
}

\author{
(1) KLAUS GOESCHEN ${ }^{1}$, (1) BERNHARD LIEDL ${ }^{2}$ \\ ${ }^{1}$ Medical School of Hannover, Kvinno Center, Germany \\ 2Reconstructive Urogenital Surgery, Pelvic Floor Centre Planegg, Planegg Urology Clinic, München-Planegg
}

\begin{abstract}
Objective: The pathogenesis of chronic pelvic pain (CPP) is still incompletely understood. Generally accepted is, that soft tissue structures mutate with age, loose tension and by this can provoke CP. This raises the question, of whether the bony pelvis underlies comparable agedependent changes and if so, how this alteration has impact on the static anatomy of the pelvic floor and the suspension and supporting system. Materials and Methods: In order to answer this question we analysed the biological evolution of human beings and checked the historical literature regarding age-dependent static changes of the bony pelvis.

Results: The vertical spine position is primarily due to an angulation of the lumbar spine against the sacrum causing a lordotic curve. The upright position of human beings forces the sacrum to curve, age dependent more and more. This leads to a descent of the promontorium causing a lifting of the coccyx and the pubic symphysis. This rotation causes a flatter pelvic floor.

Conclusion: The age-dependent, now horizontal positioned pelvic floor provokes an unphysiological stretching on the soft-tissue-pelvic-floor structures causing and boosting a decompensation of the pelvic organ support and suspension system. Overstretched connective tissue, ligaments, nerves and muscles react with pain.
\end{abstract}

Keywords: Chronic pelvic pain; bony pelvis; upright posture; pelvic organ prolapse; posterior fornix syndrome

\section{INTRODUCTION}

In the female organism, the pelvis is an especially vulnerable site for major, often disabling pathology, in particular, pain, bladder and bowel disorders. Dysmenorrhea, uterine fibroids, cycle disorders, immovable retroflexed uterus, endometriosis, inflammation of ovaries or fallopian tubes, ovarian tumour, vaginal or uterine prolapse, are all implicated in the causation of pelvic pain. ${ }^{1}$ This explains, why pelvic pain is a common clinical entity among women. Approximately 39\% of the female population reports always, often or sometimes having pelvic pain. Fifteen to twenty percent of these women are experienced in having pelvic pain longer than 1 year and $61 \%$ of them report, that the cause of their pain is unknown. ${ }^{2}$ Due to the numerous possibilities of pelvic pain sources the definition of chronic pelvic pain (CPP) is still incongruent and ambiguous in the literature. An extract and combination of the learned society definitions can be summarised as follows: CPP syndrome (CPPS) as a persistent pain in structures related to the pelvis, in either men or women, is often associated with negative cognitive, behavioural, sexual and emotional consequences, as well as with symptoms and signs related to lower urinary tract, sexual, bowel, pelvic floor or gynaecological dysfunction (European Association of 
Urology). ${ }^{3,4}$ CPP is pain in the pelvic area, that lasts for 6 months or longer (American College of Obstetrics and Gyneacologists). ${ }^{5}$ The CPPS is the occurrence of CPP, when there is no proven infection or other obvious local pathology, that may account for the pain. It is often associated with negative cognitive, behavioural, sexual or emotional consequences, as well as with symptoms suggestive of lower urinary tract, sexual, bowel or gynaecological dysfunction (International Continence Society). ${ }^{6,7}$ Even today numerous CPPS-conditions are still deemed to be of unknown origin, therefore classified as "neurological" or in the German literature as "Pelvipathia vegetativa", "Parametropathia spastica", "Spasmophilia genitalis" "Plexalgia hypogastrica", "pelvic neuralgia" or cervical syndrome". In contrast to this, 1938 Heinrich Martius already stated, that in about $30 \%$ of cases, backaches are attributed to organic factors, provoked by damaged suspending or supporting ligaments of the pelvic organs. ${ }^{9}$ Unfortunately, Martius's concept has remained largely unknown in the English literature until it was recently rediscovered. ${ }^{1}$ Independently from Martius, in 1993 Petros and UImsten ${ }^{10}$ described CPPS as being caused by lax uterosacral ligaments (USL) as part of the "Posterior Fornix Syndrome", along with other pelvic symptoms, nocturia, urgency, abnormal emptying. They reported a significant cure rate of CPPS and other posterior fornix symptoms following repair of the USL. By this they were able to verify their thesis, that insufficient ligaments can be one important origin of CPPS. Meanwhile numerous publications confirm Martius fundamental ideas and Petros data. ${ }^{1,11-25}$ Even if more and more explanations for the cause of CPPS arise, the pathogenesis of CPPS is still incompletely understood. Generally accepted is, that soft tissue structures mutate with age, loose tension and by this can provoke pelvic pain. ${ }^{1}$ This raises the question, of whether the bony pelvis underlies comparable age-dependent changes and if so, how this alteration has impact on the static anatomy of the pelvic floor and the suspension and supporting system.

\section{MATERIALS AND METHODS}

In order to find further important explanations for pelvic organ prolapse (POP) related pelvic pain we analysed the biological evolution of human beings and checked the historical literature from the static view of the bony pelvis. ${ }^{26}$

Ethics committee approval and informed consent is not necessary, because no patients involved in the study.

\section{RESULTS}

Over the long course of biological evolution the human pelvic spine, interestingly, underwent only minimal erections for the necessity of upright walk. However, this apparent little anatomical alteration allowed the human beings to change their movement from quadrupeds to bipeds, unfortunately followed by new orthostatic and pelvic floor problems. The vertical spine position is primarily due to an angulation of the lumbar spine against the sacrum causing a lordotic curve (Figure 1). The pronounced lordosis and the promontorium provide quasi "the selling purchase" for the upright position.

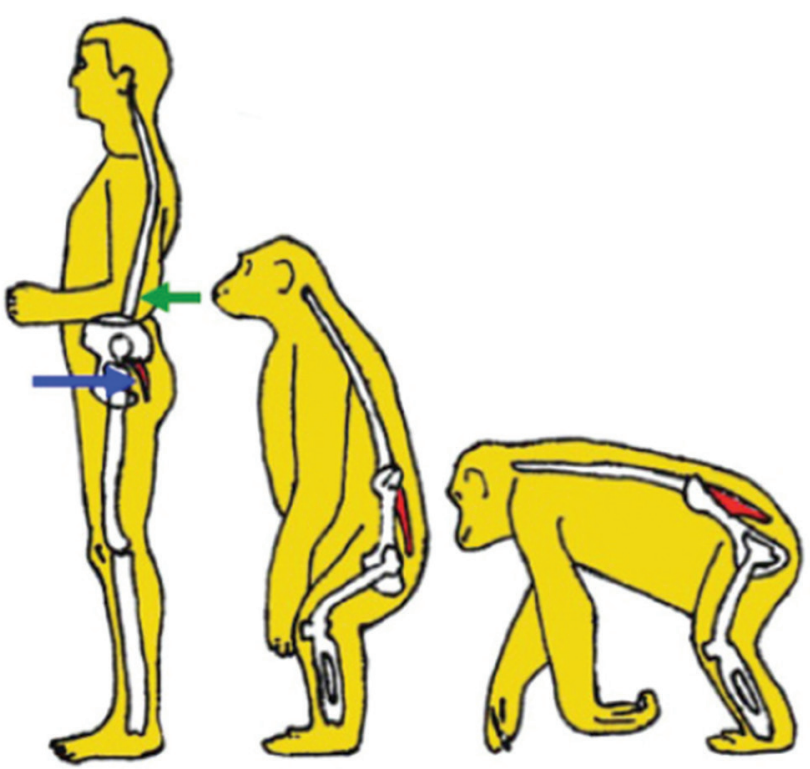

Figure 1. Change of the pelvic spine and sacrum formation from monkey to homo sapiens during biological evolution: for upright position the sacrum has to move backwards (blue arrow), the promontorium and the lumbar spine forwards (green arrow) resulting in a lordotic shape

In 1930 Heinrich Martius has already dealt with the subject pelvic spine und prolapse induced pelvic pain. He pointed out, that in women the promontorium - the base for the trunk weight - is not that strong as in men. On behalf of birth process women have to renounce on greater static strength. Therefore, the frequent sacral and back pains in women are essentially a sex specific gynaecological and orthostatic problem with equal frequency. ${ }^{27}$ Why are anatomical alterations important for prolapse, bladder/ rectum problems and pelvic pain formation? Besides deliveries, genetic predisposition, age dependent tissue atrophy, lack of oestrogen, previous operations, there are further crucial important anatomical changes responsible for symptomatic POP in the course of natural progressing life. The biped upright position of human beings forces the sacrum to curve, age dependent, more and more. This leads to a descent of the promontorium causing a lifting of the coccyx and the pubic 
symphysis. These for the formation of symptomatic POP and pelvic pain important anatomical alterations were already mentioned in the past, i.e. 1947 by Walter Stoeckel, Director of the gynaecological department of the Charité Berlin 1926-1951. In his textbook he stated, that the distance of the horizontal to the vertical plane (promontorium to upper pubic symphysis rim) consequently decreases from $9-11 \mathrm{~cm}$ to $4-5 \mathrm{~cm}$ over decades (Figure 2). This rotation causes a minor pelvic inclination with a flatter pelvic floor.

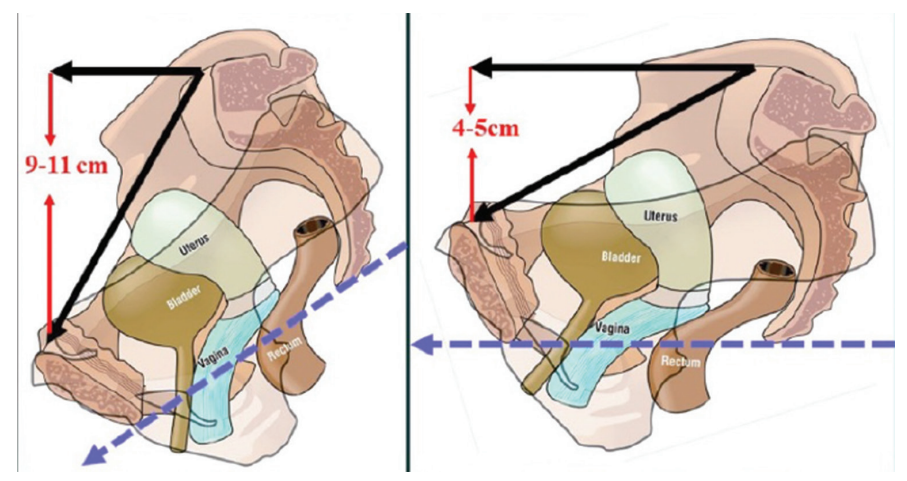

Figure 2. Natural rotation of the female pelvis during life

Left: normal female pelvis in standing position. The distance between horizontal and vertical plane (black arrows) varies between 9 and $11 \mathrm{~cm}$. The direction of the pelvic floor is approximately $45^{\circ}$ (blue dotted arrow).

Right: senior female pelvis in standing position. Due to downwards rotation of the bony pelvis the promontorium/sacrum is de- and the pubic symphysis ascended. The distance between horizontal and vertical plane (black arrows) varies only between 4 and 5 $\mathrm{cm}$. The direction of the pelvic floor (blue dotted arrow) is nearly horizontally $\left(90^{\circ}\right)$

The now much larger opening angle between conjugata anatomica and sacrum plate (Figure 3) provoke an unphysiological stretching of the soft-tissue-pelvic-floor structures causing and boosting a decompensation of the pelvic organ support and suspension system. The static system is coming more and more under pressure. However, if pressure becomes stronger, than overstretched connective tissue, ligaments, nerve fibres of the pelvic plexus and muscles react with pain., ${ }^{1,28}$ As the pelvic plexus contains sympathetic (Th12-L2), parasympathetic (S2-S4) and somatic nerves (\$2-S4) a large spectrum of different pain qualities and locations can arise, such as pelvic pain, lower abdominal, lower back ache and even pain in the vulva-region. These anatomical changes one has to keep in mind in order to understand the complex range of pelvic pain sources. It gives a very logical important explanation for the hypothesis, that CPPS has mainly an anatomical origin influenced by genetic, gender, birth, age.
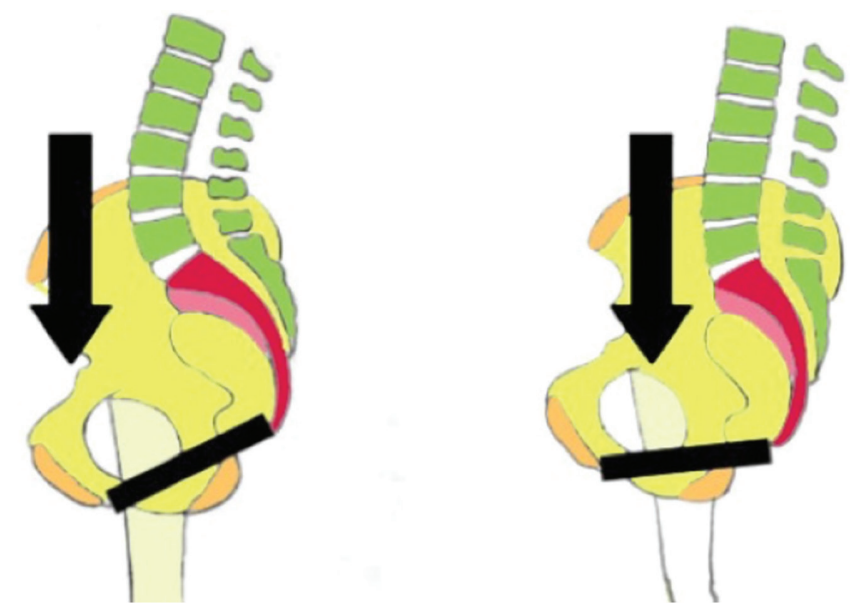

Figure 3. The inclination angle of the pelvis and thus of the pelvic floor determines the acting forces on it. Left: Normal position. Right: The more the sacrum curves, the more the pelvic floor gets a horizontal shape and comes under pressure

\section{DISCUSSION}

The aim of this article is not to list all the numerous already published causes for pelvic pain. It entirely concentrates on the very important, up to now in this context not mentioned explanation for age dependent changes in the bony static and the consequences thereof.

There are four simple mechanical reasons regarding the fact, that pelvic organs leave their normal position causing tension on the supporting and suspending system and pelvic pain: 1) Due to the upright posture of humans the pelvic organs are exposed to the effects of gravity. Therefore, the pelvic organs are pre-destinated to fall down. 2) The biped upright position of humans forces the sacrum to curve, age dependent, more and more. This leads to a descent of the promontorium causing a lifting of the coccyx and the pubic symphysis. 3) The inclination angle of the pelvis and thus of the pelvic floor is getting flat with age. The more the pelvic floor curves horizontally, the more the suspension system comes under pressure and can give way. 4) The fixation of the genital organs has to be so flexible that the tremendous change of uterine position during pregnancy and birth is possible. The pelvic floor has two functions: Firstly to obturate the abdominal cavity downwards and secondly to assure an exit for the intraabdominal organs. The fact that the outlet tract from bladder, rectum and uterus corresponds to the direction of gravity due to posture requires a particular well coordination of the suspension and support system, especially as this system still has to work properly after deliveries. The content of the small pelvis - uterus, bladder, rectum, intestine and mesenterium - is suspended and supported by muscles, ligaments and fascias. The pelvic floor contains three muscle layers located roof tile shaped one above the other. 
The upper layer, the levator ani muscle, consists of three parts: anteriorly: pars pubica, centrally: pars ischiadica, posteriorly: pars coccygea. The levator ani muscle contracts horizontally, back- and forwards against the pubourethral ligaments in front and the USL in the back.

The middle layer is to secure the hiatus genitalis, the pathway for urethra, vagina and anus, consisting of the urogenital diaphragm and the longitudinal muscle of the anus. The external layer anchors the organs consisting of the muscles: ischiocavernosus, bulbocavernosus, transversus perinei superficialis and sphincter anus externus. The levator ani is a dynamic muscle with unique physiological features, that are integral in the maintenance of urinary and faecal continence. ${ }^{29}$ The striated muscles of the levator ani differ from most other skeletal muscles in that they; 1. maintain constant tone, except during voiding, defecation, and the Valsalva manoeuvre, 2. have the ability to contract quickly at the time of an acute stress, such as a cough or sneeze, to maintain continence and 3. are able to distend considerably during parturition to allow the passage for the child and then contract after delivery to resume normal function. Despite the importance that the levator ani has for the daily well-being, it is among the most neglected and least understood parts of the human body. ${ }^{30}$ Abnormalities in the structure or function of supporting muscles and suspending ligaments can be responsible for the development of POP, sexual dysfunction, CPP, voiding and defecatory dysfunction, and urinary and faecal incontinence. Therefore, from the evolutionary perspective, it is logical, that nature additionally secures the mechanical strength of the muscular pelvic floor with fascias and ligaments in the upright walking human being. For this supplemental support an elastic system of visceral innervated muscle-connective-tissue-plate was created, the so-called lissomuscularfibrous system or endopelvic fascia. This unit is closely connected with skeleton muscle fibres, sealing interstices and is acting as a rubber mat. ${ }^{1}$ The so constructed pelvic floor represents the supporting buttress for the pelvic organs. A rupture in this fundament or an insufficiency due to tissue overstretching or connective tissue deficiency will cause a significant disturbance in the interaction of the abdominal muscle capsula formed by the pelvic floor, the abdominal wall and the diaphragm. In case of an instable pelvic floor, pressure from the abdominal wall and diaphragm cannot be captured by a sufficient counter pressure. Intestine and pelvic organs begin unbalanced to slide and get pressed down from forces acting from above. Furthermore, a weakened ligament will cause weakened muscle force, because the whole system is interrelated and ultimately reliant on firm suspensory ligaments. A striated muscle only contracts optimally over a short length. Lengthening the muscle results in a rapid loss of contractile force $=$ Gordons law. ${ }^{31}$ This can deteriorate the muscle forces enabling closure and emptying of the bladder and ano/rectum with the consequence of urinary and/or faecal incontinence and/or obstructive emptying of bladder and/or rectum. These dysfunctions frequently coexist in women with POP-induced CPPS. $3,7,9,18,23$ Age related asthenia in combination with postpartal distension of the abdominal muscles and childbirth induced damage of the pelvic floor lead consequently to enhanced muscle tension, strain on muscle insertion and suspension ligaments, hanging bellies, pronounced lordosis, high strengthening of the back muscles and lastly to a static-dynamic decompensation. ${ }^{28}$ Besides birth traumata, age dependent atrophy, genetic predisposition and previous operations the axis of the pelvic floor plays a major, so far in the literature little noticed key role regarding etiology of symptomatic vaginal and uterine prolapse and pelvic pain. An age-dependent horizontal shaping pelvic floor becomes more and more under pressure from the abdominal organs above and the gravity below. This leads in a high percentage to a decline of pelvic floor followed by a descent of pelvic organs. The unavoidable tension against the suspending ligaments can generate pain in the whole pelvic girdle. This explains, why CPP can be located overall in the pelvic area and why the frequency of pain increases with age.

\section{CONCLUSIONS}

The upright position of human beings forces the sacrum to curve, age-dependent. The more the sacrum curves, the more the pelvic floor comes in a horizontal position. The now much larger opening angle of the pelvis provoke an unphysiological stretching of the soft-tissue-pelvic-floor structures causing and boosting a decompensation of the pelvic organ support and suspension system. However, if pressure becomes stronger, than overstretched connective tissue, ligaments, nerves and muscles react with pain.

\section{ETHICS}

Ethics Committee Approval: Ethics committee approval is not necessary, because no patients involved in the study.

Informed consent: Informed consent is not necessary, because no patients involved in this study.

Peer-review: Externally peer-reviewed.

\section{Conflict of interest}

Klaus Goeschen states that he has no conflict of interest. Bernhard Liedl has received honoraria for lectures and pelvic floor workshops from American Medical Systems and Boston Scientific, honoraria for data collection (Propel study) from American Medical Systems. 


\section{Author's participation in the manuscript}

Klaus Goeschen: Project development, data collection, manuscript writing

Bernhard LiedI: Project development, manuscript writing

\section{REFERENCES}

1. Goeschen K. Role of uterosacral ligaments in the causation and cure of chronic pelvic pain syndrome. Pelviperineology 20145; 34: 2-20.

2. Mathias S, Kuppermann M, Liberman R, Lipschutz R, Steege J. Chronic pelvic pain: prevalence, health-related quality of life and economic correlates. Obstet. Gynecol 1996; 87: 321-7.

3. Engeler D, Baranowski AP, Borovicka J, Cottrell A, Dinis-Oliveira P, Elneil S, et al. (2014) Guidelines on Chronic Pelvic Pain. European Association of Urology. https://uroweb.org/wp-content/uploads/26Chronic-Pelvic-Pain_LR.pdf

4. Fall M, Baranowski AP, Elneil S, Engeler D, Hughes J, Messelink EJ, et al. (2010) EAU guidelines on chronic pelvic pain. Eur Urol 2010; 57: $35-48$.

5. Abrams P, Cardozo L, Fall M, Griffiths D, Rosier P, Ulmsten U, van Kerrebroeck $P$, Victor A, Wein A. The standardisation of terminology of lower urinary tract function: report from the Standardisation Sub-committee of the International Continence Society. Am J Obstet Gynecol 2020; 187: 116-26.

6. Abrams P, Cardozo L, Fall M, Griffiths D, Rosier P, Ulmsten U, et al. The standardisation of terminology of lower urinary tract function: report from the Standardisation Sub-committee of the International Continence Society. Neurourol Urodyn 2002; 21: 167-78.

7. Zetkin M, Schaldach H. Parametropathia spastica In: Zetkin M, Schaldach H (ed) 2002; Lexikon der Medizin. München: Elsevier

8. Martius H. Über einen häufigen gynäkologischen Symptomkomplex. Archives of Gynecology and Obstetrics. 1938; 166: 332-5.

9. Petros P, Ulmsten U. The posterior fornix syndrome: a multiple symptom complex of pelvic pain and abnormal urinary symptoms deriving from laxity in the posterior fornix. Scandinavian Journal of Urology and Nephrology. 1993; 27: 89-93.

10. Petros P. Severe chronic pelvic pain in women may be caused by ligamentous laxity in the posterior fornix of the vagina. Aust. NZ. J. Obstet. Gynaecol. 1996; 36: 351-4.

11. Petros P. New ambulatory surgical methods using an anatomical classification of urinary dysfunction improve stress, urge, and abnormal emptying. Int J Urogynecol 1997; 5: 270-8.

12. Farnsworth BN. Posterior intravaginal slingplasty (infracoccygeal sacropexy) for severe posthysterectom vaginal vault prolapse: a preliminary report on efficacy and safety. Int. Urogynecol. J. Pelvic Floor Dysfunct. 2002; 13: 4-8.

13. Goeschen K, Gent HJ. Das posteriore fornix syndrom. Frauenarzt 2004; 45:104-12.
14. Neuman M, Lavy Y. Posterior intra-vaginal slingplasty for the treatment of vaginal apex prolapse: medium-term results of 140 operations with a novel procedure. Eur J Obstet Gynecol Reprod Biol 2008; 140: 230-3.

15. Petros P, Richardson PA. TFS posterior sling improves overactive bladder, pelvic pain and abnormal emptying, even with minor prolapse: a prospective urodynamic study.

16. Pelviperineology 2010; 29: 52-5.

17. Müller-Funogea A. Posterior fornix syndrome: a new urogynecologic entity. Ethiopathogenesis and proposal for surgical therapy. Thesis. Romania: Medical University of Bucharest, 2014.

18. Goeschen K. Posterior fornix syndrome: comparison of original (2004) and modified (2015) post-PIVS anatomic and symptomatic results - a personal journey. Pelviperineology 2015; 34: 85-91.

19. Caliskan A, Goeschen K, Zumrutbas AE. Long term results of modified posterior intravaginal slingplasty (P-IVS) in patients with pelvic organ prolapse. Pelviperineology 2015; 34: 94-100.

20. Inoue H, Kohata Y, Sekiguchi Y, Kusaka T, Fukuda T, Monnma M. The TFS minisling restores major pelvic organ prolapse and symptoms in aged Japanese women by repairing damaged suspensory ligaments 12-48 month data. Pelviperineology 2015; 34: 79-83.

21. Liedl B, Lukban J, Stanford E, et al. Besserung von Symptomen der Stuhlinkontinenz durch Beckenbodenrekonstruktion mit elevate posterior/ apical und elevate anterior/apical. Coloproct 2016; 38 : 75-6.

22. Goeschen K, Gold DM. Application of the Pescatori iceberg to 198 patients presenting with chronic pelvic pain before and after posterior sling surgery. Pelviperineology 2017; 36: 1-4.

23. Liedl B. Goeschen K, Durner L. Current treatment of pelvic organ prolapse correlated with chronic pelvic pain, bladder and bowel dysfunction. Curr Opin Urol 2017; 27: 274-81.

24. Petros P, Goeschen K, Inoue H. Underactive bladder may be caused by uterosacral ligament laxity - a critical review of two paradigms. Cent. European J Urol 2018; 71: 449-52.

25. Liedl B, Goeschen K, Sutherland SE, Roovers JP, Yassouridis A. Can surgical reconstruction of vaginal and ligamentous laxity cure overactive bladder symptoms in women with pelvic organ prolapse? BJU International. 2018; doi:10.1111/bju.14453

26. Martius H. Die Kreuzschmerzen der Frau. Georg Thieme Verlag, 2. Aufl,1944.

27. Martius H (1930) Umformungen und andere Anomalien der Wirbelsäulen und ihre pathologische Bedeutung. Arch Gynäk 1930; 139: 335-9.

28. Stoeckel W. Lehrbuch der Gynäkologie. Hirzel, Leipzig, 1947, 11th Edition, p720-4.

29. Wester C, Brubaker L. Normal pelvic floor physiology. Obstet Gynecol Clin North Am 1998; 25: 707-22.

30. Wall LL. The muscles of the pelvic floor. Clin Obstet Gynecol 1993; 36: $910-25$.

31. Gordon AM, Huxley AF, Julian FJ. The variation in isometric tension with sarcomere length in vertebrate muscle fibres. J Physiol 1966; 184: 170-92. 